Research Article,

\title{
The TOAST (Traction on Ankle Stress Test): A Supplemental Clinical and Dynamic Ultrasound Examination Approach for Focal Assessment of Axial Ankle Joint Instability.
}

\author{
James Inklebarger ${ }^{1}$, Mr Thomas Rimbault ${ }^{2}$, Teja Joshi ${ }^{3}$, Kathy Whitehouse ${ }^{4}$ \\ ${ }^{1}$ Director, London Prolotherapy \\ ${ }^{2}$ Principal Podiatrist Margaret Dabbs, London \\ ${ }^{3}$ Physiotherapist, Ravenscroft Health Centre, London \\ ${ }^{4}$ Alexander Technique practitioner, with special interest in performing arts injury rehabilitation \\ Email Address: James.inklebarger@yahoo.co.uk
}

\begin{abstract}
:
Ankle drawer and varus-valgus stress tests have evolved to examine for excessive joint displacement in the sagittal and coronal planes which may correlate to ligament instability. However, there appear to be no formal manual test(s) assessing for axial plane (longitudinal) instability of the mortise joint. In keeping with Cartesian and Cyriax principles, The TOAST (Traction On Ankle Stress Test) was developed to dynamically assess for $3^{\text {rd }}$ vector axial strain pain generation and long-axis mortise joint gapping. The test is easy to learn as longitudinal manual traction of the mortise joint and applied counterforce hand positions are similar to emergent ankle fracture dislocation reduction technique. The TOAST may also be performed under dynamic ultrasound control.
\end{abstract}

Keywords: Ankle Instability, TOAST Maneuver, Ultrasound Dynamic Stress Test, Traction.

\section{Introduction:}

Around $20 \%$ of acute ankle sprain patients develop sequella of chronic ankle instability, with the failure of functional rehabilitation cited as causal. Differentiation between functional and anatomical ankle instability is very essential to guide the proper treatment, with current stability tests including the varus stress test and drawer tests recommended. ${ }^{2}$

Chronic mechano-ligamentous insufficiency particularly of the anterior talofibular ligament (ATFL) commonly follows ankle inversion injury. Instability occurs when ligaments rupture, or fail to remodel to normal length, allowing motion beyond normal physiological limits. The ankle joint capsule and soft tissues about the joint may also be compromised, confounding rehabilitation of proprioceptive innervation and functional instability. 1

Current ankle stress tests assess stability in sagittal and coronal planes. The TOAST maneuver was developed for a $3^{\text {rd }}$ vector assessment of occult longitudinal ankle mortise joint stability.

\section{METHOD-Figure 1:}

1. The table height is adjusted with adequate hind floor space for the examiner to lean back and adopt body positions for force generation efficiency. (Image 1)

2. The patient is positioned supine on the examining table and asked to grip the table during the test, or the knee countertraction is applied by an assistant.

3. TOAST is first performed on the uninvolved side to establish the baseline maximum mortise joint end of range gapping

4. The TOAST is performed by first lifting the knee straight lower limb off the table holding while firmly-cupping and supporting the posterior aspect of the heel, with palm and 

Examination Approach for Focal Assessment of Axial Ankle Joint Instability.

fingers wrapping around and calcaneum. (Images $2 \& 3$ )

5. The ankle is held in a neutral position. (If the examiner is small of stature or body mass, the elbows are flexed to 90 degrees and held tight to the ribcage.

6. Hand positions: The distal aspect of the preferred volar forearm is wrapped perpendicularly around the forefoot, just below the anterior mortise joint line.) The fingers of the other hand the wrap around the medial aspect of the forefoot. (note: The hand positions are reversed for examining the other ankle.

7. Maintaining a 2-handed grip with equal pressure, the ankle joint is slowly and gradually tractioned and then slowly, gradually and released in a controlled pulse manner over a few seconds. The test may be repeated several times to fully appreciate end range gapping, joint play, and feel for potential mortise subluxation-relocation, axial joint laxity, and crepitus.

8. In cases of marked ankle mortise longitudinal laxity the anterior mortise joint may be also noted to visually gap at the end of range traction.

9. The TOAST may also be performed under dynamic (US) ultrasound (image 4) or fluoroscopic guidance. If US is used, a linear probe is recommended with long axis

Orientation over the mid-line anterior joint. The forefoot may need to be slightly plantar flexed for better probe contact. Bilateral image capture at end of range of maximal axial traction is recommended for documentation regardless of method, with asymmetric measurements provides further objective verification of comparative axial mortise joint instability. If Fluoroscopy is used, the operator should wear lead gloves.

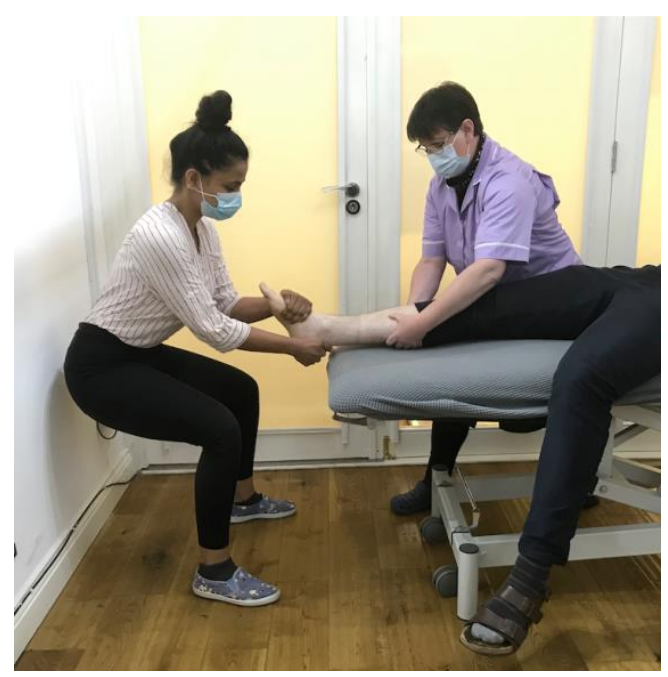

Figure 1: Operator TOAST body \& hand positions an with assistant applying counter traction
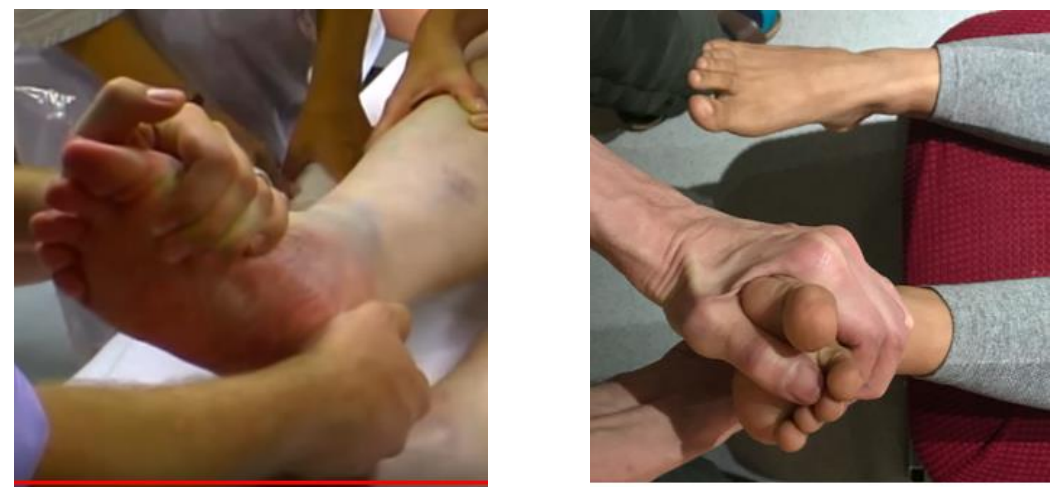

Figure 2 \& 3: TOAST with pulsed or progressive traction applied in the superior to inferior axial plane followed by a gradual release. Unilateral crepitus, excessive axial joint play, and reduction mortise click-clunk with our without pain are indicators of a positive test. 

Examination Approach for Focal Assessment of Axial Ankle Joint Instability.

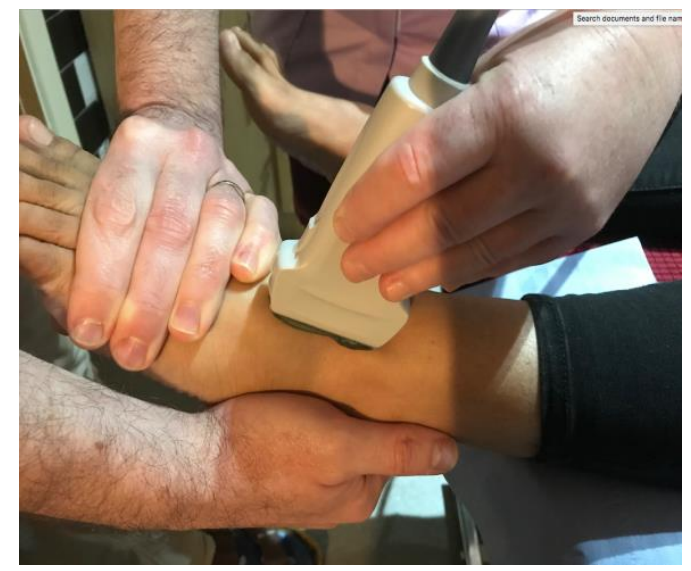

Figure 4: TOAST under ultrasound
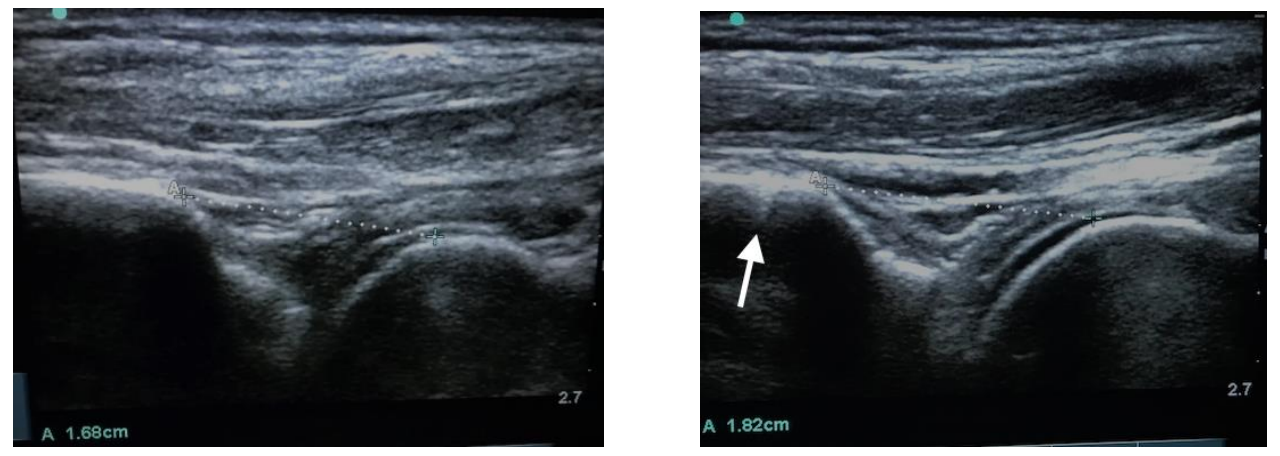

Fugure: 5 \& 6: Right \& left ankle TOAST images, demonstrating a relatively greater left ankle mortise joint widening (13mm) on the chronically unstable left. Also note the appearance of incidental cortex irregularity at the left distal tibial plafond (white arrow), which may be in keeping with an old Salter Harris type 1 fracture.

\section{Discussion}

By applying Cartesian principles to mortise joint examination, a $3^{\text {rd }}$ supplementary longitudinal examination vector may enhance the evaluation of ankle joint stability, particularly in patients reporting ankle Joint pain, perceived instability, and recurrent low-threshold ankle inversions, and hypermobility syndromes.

This $3^{\text {rd }}$ source approach is also common in STEM (Science Technology, Engineering Mathematics,

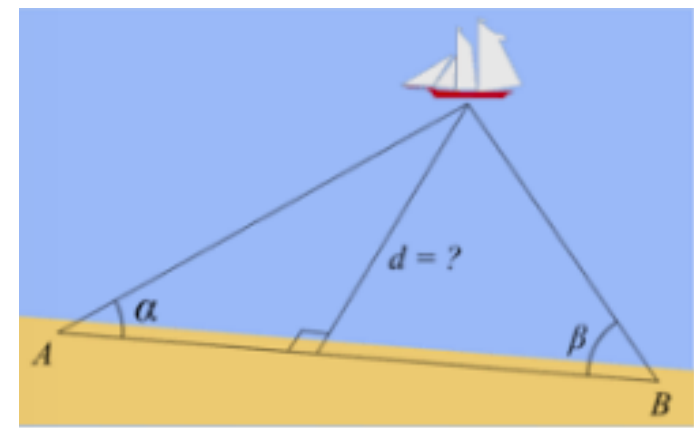

Figure 6
Navigation) fields, which also find value in source verification by at least three credible, independent yet complimentary data streams.

It may therefore be useful to add this $3^{\text {rd }}$ axial vector to ankle joint stability examination in order to supplement existing anterior drawer (sagittal) and coronal plane (varus-valgus) stress tests. (Images 8-10)

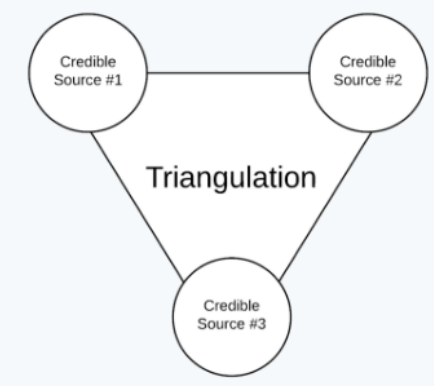

Figure 7 


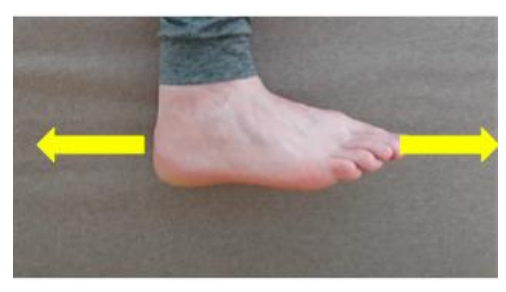

Figure 8

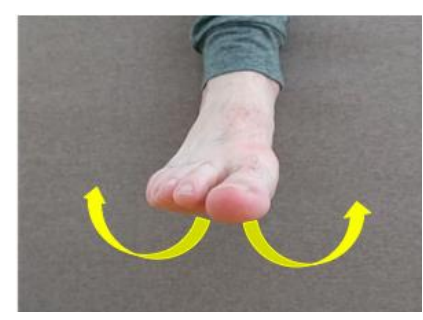

Figure 9

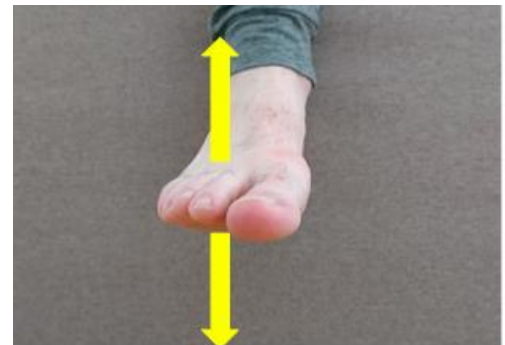

Figure 10

Figure 8-10 Left to Right: Sagittal plane (Axis for the Anterior-Posterior Drawer test), Coronal plane: (Axis for the Varus-Valgus Talar Tilt test), Longitudinal plane: Axis for the (TOAST) Traction on Ankle Stress

It is thought that axial instability may also occur in isolation and might be missed if not tested. There may also be concomitant findings of clinician and patient- perceived audible joint crepitus, palpable clunks, and mortise joint subluxation-relocation which in some cases may even be visible on applied longitudinal traction and release.

Though lower grade ankle sprains may typically involve the ATFL, more serious injuries such as high grade sprains and fracture-dislocations may affect other supporting integrity structures. Coupled force vectors in higher grade injuries or chronic low threshold sprains may also have a potential for concomitant long axis instability and involve the ankle joint capsule. The ankle Anterior Drawer (ATFL) and ankle abduction-adduction Eversion-Inversion Talar Tilt (CFL-Deltoid ligament) tests are both pain provocativeinstability stress tests for detecting excessive joint play in sagittal and coronal planes. However, there is currently no stress tests documented in the literature, for assessing axial integrity of the mortise joint.

The TOAST was developed as a clinical application of René Descartes triangulation XYZ vector theories, ${ }^{3}$ and evolved by the as a means of assessing for the thus far missing $3^{\text {rd }}$ vector of long axis mortise joint instability. The TOAST traction hand positions are also not original, being derived from emergency ankle fracture dislocation reduction maneuvers. 4

\section{Discussion:}

In this example, there was a wider symptomatic side left mortise joint gap at end of range traction.

The structures provided axial stability to the mortise joint include the CFL and to a lesser extent the ATFL and PTFL, the Deltoid ligaments, as well as the medial and lateral tendon complexes and the joint capsule itself. The TOAST test traction and counter traction hand positions are similar to those of ankle fracture-dislocation reduction. 5 TOAST may also be uniquely pain generating and identify axial instability in isolation, when the traditional drawer and varusvalgus stress tests are negative.

\section{Conclusion:}

The TOAST maneuver performed with or without ultrasound, may provide a supplemental and uniquely isolating $3^{\text {rd }}$ longitudinal ankle joint stability testing vector, which may not readily be apparent with existing ankle stability tests.

However, the aim is not to replace, but rather complement The TOAST performed under dynamic ultrasound may also add an element of quantification. The test appears to be simple to learn with thus far satisfactory intra-examiner reproducibility and patient tolerability. However, further studies will be required to define this tests limitations, indications, clinical utility, inter operator agreement and reliability.

\section{Acknowledgements:}

Special thanks to Tim 'Nobby' Clarke, Rachel Dembry, Julie Brumby and Sarah Jane King

\section{References:}

[1] Walls RJ, Ros KA, Fraser EJ, Hodgkins CW, Smyth A, Egan CJ, Calder J, Kennedy JG, Football injuries of the ankle: A review of injury mechanisms, diagnosis and management, World $\mathbf{J}$ Orthop. Jan 18, 2016; 7(1): 8-19

[2] Al-Mohrej OA, Al-Kenani NS, Chronic ankle instability: Current perspectives, Avicenna J Med, 2016 Oct-Dec;6(4):103-8. 
[3] Glaser, A.K., Chen, Y., Yin, C. et al. Multidirectional digital scanned lightsheet microscopy enables uniform fluorescence excitation and contrastenhanced imaging. Sci Rep 8, 13878 (2018). https://doi.org/10.1038/s41598018-32367-5

[4] Abraham A. Emergency treatment of ankle fracture dislocations - a reliable technique for early reduction. Ann R Coll Surg Engl. 2003;85(6):427. doi:10.1308/003588403322520870

[5] Choudhry B, Leung B, Filips E, Dhaliwal K. Keeping the Traction on in Orthopaedics. Cureus. 2020;12(8):e10034. Published $2020 \quad$ Aug 25. doi:10.7759/cureus.10034 